\title{
Research on Gas Insulated Switchgear Internal Fault Diagnostic Methods
}

\author{
Si Yadong ${ }^{1}$, Jia Xinmiao ${ }^{1}$, Guo Zhi ${ }^{1}$, Jia Yuliang ${ }^{1}$, Feng Tiemin $^{1}, \mathrm{Ma} \mathrm{Wei}^{2}$, Zhang \\ Yuhui ${ }^{2}$, Zhang Hong ${ }^{3}$, Cao Huiyan ${ }^{4}$, Yang Yingying ${ }^{4}$, Guan Ling ${ }^{4}$, Jiao Hongzhi' ${ }^{4}$, Gao \\ $\mathrm{Wei}^{4}$ \\ ${ }^{1}$ Chaoyang Power Supply Company, Liaoning Electric Power Company Limited, State Grid, China \\ ${ }^{2}$ Liaoyang Power Supply Company, Liaoning Electric Power Company Limited, State Grid, China \\ ${ }^{3}$ Jinzhou Power Supply Company, Liaoning Electric Power Company Limited, State Grid, China \\ ${ }^{4}$ Fushun Power Supply Company, Liaoning Electric Power Company Limited, State Grid, China
} fushunpowersupply@163.com

Keywords: GIS, Partial discharge, UHF, Ultrasound, SF6, Fault location

\begin{abstract}
The types of internal fault and their influencing factors in GIS are analyzed. The key technologies and researching results of UHF, ultrasound and SF6 decomposition method on site for GIS internal fault detecting are presented, compared with each other and analyzed. The methods of fault location and the fault type classification method are summarized finally.
\end{abstract}

\section{Introduction}

The gas-insulated metal-enclosed switchgear (GIS) has been a large number of applications in the power system by its virtue of small size, reliable operation, safety and long maintenance cycle. Although GIS has the advantage of high security and reliability, maintenance-free, but in the actual its operation is still often have different types of failure, and causes power outages by a long time, high maintenance costs, huge losses, and affects the safe operation of the power system and reliability for electricity. So GIS fault detection and diagnostic technology gets wide attention $[1,2]$.

\section{GIS internal causes of the faults}

GIS equipment in the process of manufacturing, transportation, installation and operation are likely to defect, resulting in partial discharge of GIS internal failure. The main defects are the current-carrying conductor surface burrs, sharp corners and the unreasonable design, bubbles, cracks and other defects in the casting insulators; free conductive particles of the residual metal shavings or metal particles in the device, insulator conductor at the interface of the air gap and so on. Partial discharge of a variety may lead to damage of the entire GIS [3]. Different type of defect caused by insulation failure statistics are as shown in Table 1.

Table1 Different type of defect caused by insulation failure statistics

\begin{tabular}{lc}
\hline Type of defect & Fault ratio (\%) \\
\hline Particles and foreign bodies & 28.17 \\
The main contact poor contact & 15.49 \\
Shield poor contact & 25.35 \\
Wet & 9.86 \\
The high voltage conductor protrusion & 7.04 \\
The bubble in insulator & 14.09 \\
\hline
\end{tabular}

\section{GIS fault detection methods}

At present, GIS Internal diagnostic methods applied to the scene are mainly the based on ultra high frequency (UHF), the ultrasonic method and SF6 gas decomposition products. 
(1) UHF method

UHF method is gathering firstly 0.3G-3GHZ ultra high-frequency electromagnetic signals from GIS equipment caused by internal partial discharge by the coupling antenna, then identify GIS partial discharge type according to the measured signal.

Antenna for measuring ultra high frequency electromagnetic signals can be divided into two kinds that is internal and external type. Built-in antenna in the GIS manufacturing, installation, usually installed in the GIS equipment, hand hole or the inner wall of the hatch cover, generally disc-shaped UHF antenna, the plane of the shell wall flush to minimize internal electric field distribution [4]. The sensors can directly receive the signal to an external sensing efficiency, GIS metal shell can shield external electromagnetic interference, and easy to receive electromagnetic signals generated by the GIS partial discharge. The built-in antenna structure is shown in Figure 1.

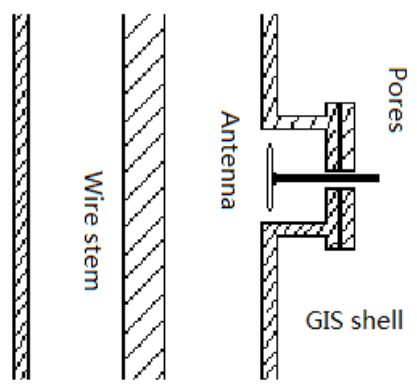

Figure 1 The built-in antenna schematic

The external antenna is placed between the GIS metal casing and basin insulator connecting flanges, receiving the electromagnetic waves that spread out from there. External antenna structure is shown in Figure 2. The advantage is using flexibly and conveniently, easy to move and not the demolition of GIS. The center frequency of the antenna should avoid air corona interference, while taking the shielding measures. In order to meet the requirements of sensitivity and noise immunity, generally uses coaxial waveguide sensors, slot coaxial waveguide sensors and the slot antenna. Sensitivity of Coaxial waveguide sensor is equal to or higher than the built-in disc antenna [5].

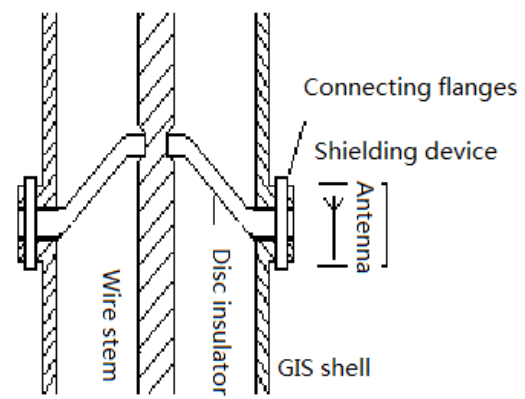

Figure 2 The external antenna schematic

(2) Method of the ultrasonic

When in GIS internal occurs Pd and other fault, ultrasound will be emitted, this ultrasound get through SF6 gas and GIS case and be weakened, but still can be detected by the piezoelectric element outside of the GIS. Due to the relationship of the ultrasonic signal and the PD level is essentially linear, so $10 \sim 100 \mathrm{kHz}$ ultrasound signal can be received through the ultrasonic sensing probe. These ultrasonic detection and analysis can be drawn to the intensity of the acoustic signal, the frequency components, frequency and power frequency correlation information and the original waveform and the phase map of the sound signal, according to the signal characteristics can be fault diagnosing.

The method is non-destructive testing, site test equipment need not outage, don't need other auxiliary equipment and facilities; Have a good scene anti-interference performance; the drawback 
of the ultrasound method is very easy to judge defective or not, but the judgment of the defect type and severity analysis rely on experience.

(3) $\mathrm{SF}_{6}$ gas decomposition test

Internal fault state of GIS equipment generally has a floating potential discharge, corona discharge, arc discharge, spark discharge, and thermal decomposition and so on, SF6 gas and solid insulating materials will be generated the fluoride and sulfide by the decomposition in the fault area.

When SF6 electrical equipment is faulty, the fault area SF6 gas and solid insulating materials cracking in the role of heat and electricity will produce sulfide and fluoride. Sulfide mainly includes $\mathrm{SO}_{2}, \mathrm{H}_{2} \mathrm{~S}, \mathrm{SF}_{4}, \mathrm{SO}_{2} \mathrm{~F}_{2}$ and $\mathrm{SOF}_{2}$; fluoride mainly includes $\mathrm{HF}, \mathrm{CF}_{4}$ and metal of fluorides. Through the detection the existence and content of the two characteristic components of $\mathrm{SO}_{2}$ and $\mathrm{H}_{2} \mathrm{~S}$ in $\mathrm{SF}_{6}$ gas, to aid in failure analysis.

\section{Partial discharge location measurements}

(1) UHF method of positioning measurement

Partial discharge location main in accordance with the jet lag of the pulse signal the different of the detection points, according to the propagation speed of the jet lag and the electromagnetic wave to locate. So to solve two problems: First, the propagation velocity of the PD signals in GIS internal; Second, the starting time of the PD.

Through the UHF signal is measured by internal or external sensor, the fault can be location. But compared with the GIS internal fixed installed sensors, external sensors can be constantly changing measurement point location, has great flexibility in terms of positioning measurement. The following are main methods based on external positioning measurement.

1) Comparison of signal amplitude method

Sensors turn on each disc insulator and compare the size of detection signal; the location of disc insulators with the biggest signal is close to the discharge source location throughout. This can rough positioning the discharge power.

2) Split-face method

When the discharge signal exists in prodigious space, we can free to choose a position, alone put two sensors the A and B, and adjust their position so that the signal time lag is zero, it shows that the discharge power is at the split-face P1 of A and B. Change the orientation, do the same measurements, and get different split-face P2 and P3. The intersection of these split-faces is the location of the discharge power. Method of split-face shown in Fig. 3

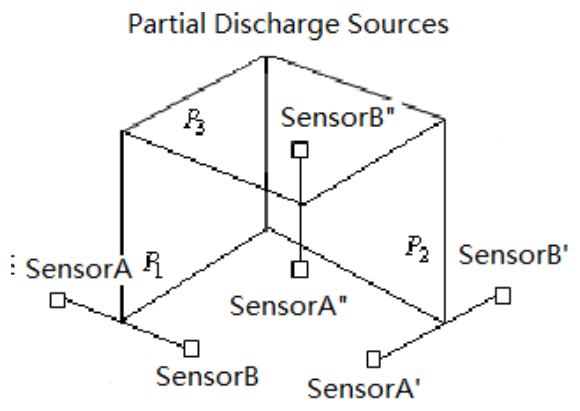

Figure 3 The split-face positioning method schematic

3) Signal comparison method

Two sensors are placed in the adjacent disc insulator or nearby spatial location, through observe the reach order of two sensor signals, we can determine that the discharge source close to the signal leading sensor. By moving the two sensors, ultimately determine the location of the discharge source

4) two-point time lag calculation

If you need to determine the location of the discharge source between the two disc insulators, two of sensors can be used to accurately measure the signal time lag of the two disc insulators, and then 
calculate the location of the discharge source according to the propagation velocity of electromagnetic waves

(2) Ultrasound measurement locate method

1) Time lag estimation

Compared to UHF positioning time lag measurement method, the same, through comparing the measurement signal time lag of different points, the fault location will be determined. Theoretically, the sound propagation speed is much slower than electromagnetic waves; the measurement of time lag is easier, so positioning will more accurate. But signal acquisition need more than one sensors, positioning accuracy is not high in the field of strong electromagnetic interference.

2) Amplitude comparative method

The most simple and practical method in the ultrasonic positioning is based on signal amplitude variation method, the sensor near the sound source, the measured signal amplitude is large, and vice versa, the signal amplitude is small.

Such as the same long bus and no high-reflection structure, sensors which moving on the shell, if the signal maximum value in a wide range can be identified the failure on the center conductor; if the maximum value at a specific point, the failure can be determined on the shell.

\section{Conclusions}

UHF and ultrasonic methods are applicable to the GIS partial discharge detection. They have very good sensitivity. The UHF method suitable for GIS of the continuous monitoring, acoustic emission method is suitable for online testing of phases. At present, it is not a clear relationship between signal strength and heavy volume of the Bureau by measuring of Ultrasonic method and UHF method the measurement. The above two methods and SF6 gas decomposition test method complement each and apply to GIS fault diagnostic field may achieve better results. Both UHF and ultrasonic methods all can measure the fault location and identify the type of faults.

\section{References}

[1]Zhang Xing, Yao Yao, Tang Ju, et al. Actuality and Perspective of proximate Analysis of SF6 Decomposed Products under Partial Discharge [J]. High Voltage Engineering, 2008 (4): 664-669

[2]Fang Qing, Liu Weidong, Tang Wei, et al. A New Ultrasonic Diagnosis Technology for Internal Default of GIS Equipment[J]. High-voltage Apparatus, 2008, 44 (6): 589-591

[3]Qiao Wei, Feng Yunping. GIS Partial Discharge Detection and Recognition [J]. High-voltage Apparatus,, 1995, 5: 37 -41

[4]Yinhua Yuchang, Zhang Xiaoyong, et al. UHF Signal Extraction for GIS Partial Discharge Detection [J]. High-voltage Apparatus, 2004,40 (5): 330-332

[5]Mizojiri, T. et al. Sensitivities and noise-resistant performances of various PD sensors located outside a GIS. International Symposium on Electrical Insulating Materials,2008. 2008,9: 573 - 576 